\title{
Progress and recent trends in generative design
}

\author{
Ioannis Ntintakis ${ }^{1,2}$ and George E. Stavroulakis ${ }^{1, *}$ \\ ${ }^{1}$ School of Production Engineering and Management, Computational Mechanics and Optimization \\ Laboratory, Technical University of Crete GR-73100 Chania, Greece email: gestavr@dpem.tuc.gr, \\ www.comeco.tuc.gr \\ ${ }^{2}$ School of Engineer, Department of Mechanical Engineering, Hellenic Mediterranean University, \\ Estauromenos, 71004 Heraklion, Greece e-mail: ntintakis@hmu.gr
}

\begin{abstract}
Due to recent developments in the field of additive manufacturing enormous advantages have become in product design and manufacturing process. Before the appearance of additive manufacturing, developing very complex or light weight structures was difficult to manufacture. The development of artificial intelligent technology helps to develop new collaborative tools and algorithms. Generative design approach is one of them. The outcome model from a generative design study is not depending only from designer/engineer experience or his knowledge. Designers can react with sophisticated algorithms through CAD programs to specify the shape and the topology of the model. A significant tool on a generative design system is topology optimization which is able to generate different solutions. The changes in design process are significant. A rough conceptual design (sketch) or a $3 \mathrm{~d}$ model is first prepared. Then, boundary conditions, safety factor, manufacturing limitations and materials properties are defined. The generative design system generates potential solutions. It's up to the designer to find the design that best fits to his need. In this paper the review covers the limitations of current systems through the study of specific design cases using commercial generative design systems.
\end{abstract}

\section{INTRODUCTION}

During the design process designers/engineers has to take decision for product shape, ergonomic design, material selection, product stiffness, durability and so on. The combination of these parameters create a complex problem. Designer has to take a decision very fast and in accurate. In this effort advanced systems like generative design, topology optimization and additive manufacturing are very helpful.

Generative Design (GD) has become more and more popular in recent years. In general GD mimics nature by using algorithms inspired, for example, by the way in which bones grow in animals. A basic outcome of this system is to add material where is need to be and to remove from the domain where it doesn't. GD is used today in mechanical design, architecture and civil engineering, microstructural design, smart structures, micromechanics, multiphysics and other [1-2]. As a term is the repetition of process of potential outcomes that meet certain constrains, boundary conditions. Designer can change the inputs in a way to

* Corresponding author: gestavr@dpem.tuc.gr 
choose the best solution. It is therefore a fast method of exploring design possibilities that is used in various design fields. As a process generative design can be analysed as a linear diagram [3]. The process starts with an idea which designer set up in the system. Mainly the idea described in a general form with a rough $3 \mathrm{~d}$ model or a sketch. Then the rules are defined and an algorithm starts to formalize the rules in parameters in order to output potential solutions. Each time the algorithm offers a number of different solutions. Designer studies the solutions and modifies the inputs rules so to take more accurate solutions. The process ends when the designer judges that the potential solution is the appropriate. The algorithmic method transform requirements into design and product.

\section{GENERATIVE DESIGN IN INDUSTRY}

In 2019 the company Kartell produced the first commercial chair made using generative design in collaboration with Autodesk and with famous designer Philippe Starck. As Starck said they ask from intelligent system to carry the body chair with the least amount of material. In aerospace industry generative design is a well know technology. Also, in RUAG Sentil satellite the designers used topology optimization and Additive Manufacturing (AM) for the antenna bracket with EOS Aluminium AlSi10Mg which has high strength and resistance to dynamic stress. The optimization goal wasn't only the light weight structure but also the minimization of undesired vibration from rocket high speed. The antenna bracket weight reduction was over than $40 \%$, from $1.6 \mathrm{~kg}$ to $0.94 \mathrm{~kg}$ [4].

In automotive sector in 2018 General Motors starts to develop new car components. One of them is a new seat bracket which is be $40 \%$ lighter and $20 \%$ stiffer than the previous one. The potential solutions was chosen from about 150 different models [5]. Audi used AM to produce the water connection for the W12 engine and Porsche makes the gearstick for the 959 model. BMW producing more over than 25,000 prototypes helping the design process [6]. Also, in Ford there is no need for tooling for prototypes saving a huge cost in product development process [7-8].

In aviation industry the Airbus group has run out an analytically study about the reducing of $\mathrm{CO} 2$ emission about $40 \%$ from weight reduction. For this purpose they redesign with TO toll the Airbus A320 nacelle hinge Bracket using the direct metal laser sintering AM technology rather than traditional casting methods [9].

\section{Soft Robotics}

Traditional rigid robots are characterized by their incredibly powerful and precise performance abilities. A typical robot construction is made of rigid materials and rigid joints. There is no ability for elastic deformation therefore in case the robot acts on rigid bodies it cannot adapt its shape. The case arises, for example, during interactions with human body or environmental constrains which can also be defined as lack of multi-functionality [10].

In the domain of healthcare, robots have to adapt their functionality in human body requirements and to be more sensitive, compliant and adaptive. Next generation robots have to be more deformable and their shape can be inspired from nature. In the design and control of new age robots biological principles are applied, the so-called soft robots. Soft robots are expected to have better adaptability on human constrains with safe and smooth interaction with them [11].

A typical structure of a soft robot consists from a number of actuators which are usually manufactured from deformable material. Usually actuators activated with compressed air thus generate motion [12]. In other cases fluid motion creates mechanical action [13]. Other researcher [14] gives a specific spatial architecture to the bladder as a bellow. Or other use 
thin films for the bladder structure [15]. The film flexibility and the appropriate plane stiffness conduce to transform the structure in the right way. The use of materials with different stiffness values can easily be used in order to add 'programming' to the behaviour of the bladder. The use of stiffer material in only one side of the actuator is another possibility [16]. Other actuators allow faster actuation with lower volume change. Along the surface of each finger flexible tubes are established, so allowing faster actuation. A soft robot is composed of three parts, an actuator, a sensor(s) and a controller.

In this study we are going to test an actuator which is powered by air. The aim of this series of experiments was to evaluate the inflating behaviour of a soft robotic actuator using materials with different stiffness so on to add a programming behavior to the actuator. Soft robots mimic the nature and especially animal capabilities and they are at the crossroad of engineering and mechatronics, chemistry and plastics [17]. Also we are going to use a generative design platform in order to design a rigid body to reinforce a soft robot actuator. The new design stability will be tested by using a FEA analysis which is the appropriate tool to test a wide range of structures [18]. Results related to the design of controlled flexible structures can be founr in [19-20]

\section{Generative Design (GD) and Topology Optimization (TO)}

Topology Optimization is a basic tool in generative design. In GD potential solutions refer to different size and shape of the product. TO results combine shape and size in order to satisfy the objective function which usually wants to minimize the volume [21]. So designers using topology optimization are able to select a design solution that satisfy design requirements. Very often the selected solution is not manufactured or it is economically unprofitable with subtracted methods.

In particular TO has the following benefits in the design: a) creating light structures b) generation of a ready-to-build part/assembly c) minimize the amount of raw material d) energy saving e) less need for natural prototypes f) reduction of physical testing g) reduced entry time [22]. Elastic properties of a material compared with their density may vary in model area. The material can be permanently removed from model space. During the TO study models have to be applied all necessary boundary conditions. Two methods have been developed for TO study. The first one is truss based and second is volume based.

The meaning of optimization problem is a combination of many parameters. The target is to find the appropriate structural design solution in a domain. To solve the optimization problem restrict parameters as set up, such as, the boundary conditions, the initial volume and the specific area which will remain void or will be filled with material. Potential solutions for the shape or the size of the model are unknown [23]. Mathematical formulation is specified from minimize of the objective function specified as below:

$$
\begin{aligned}
& \text { Specify } \chi=\left\{\begin{array}{c}
\chi_{1} \\
\chi_{2} \\
\cdot \\
\cdot \\
x_{n}
\end{array}\right\} \text { which minimize } \mathrm{f}(\mathrm{x}) \\
& \text { where } g i(x) \leq 0, i=1,2, \ldots, m \\
& \text { hj }(x)=0, j=1,2, \ldots, n
\end{aligned}
$$

The domain area is an n-distance Cartesian area in which each axis matched in a parameter. Where $\mathrm{x}$ is the vector of the variables that the design must meet in order to be acceptable. There are essentially two categories of restrictions: a) Operational constraints refer to system behavioral constraints b) Geometric constraints refer to physical constraints. Where, $\mathrm{f}(\mathrm{x})$ is the objective function or the cost function, essentially a criterion for comparing 
acceptable solutions to select the optimal design. The objective is to minimize $f(x)$. The functions gi (x) and hj (x) are called constraints of inequality and equality that determine the constraints of the problem. Points on the constraint surface with gi $(x)=0$ satisfy the constraints marginally, so the design area is divided into two distinct spaces. The first space refers to marginally acceptable and acceptable points where gi $(x)<0$. The second space refers to unacceptable points and applies gi $(\mathrm{x})>0$.

Extensions of topology optimization in order to take into account wished deformability patterns, and thus instead of a structure to deliver a flexible mechanism, are also possible [2324]. These results can be used for the design of innovative metamaterials with optimal microstructures, like auxetics [25].

\section{Experimental Validation}

We are going to test a common shape of a soft robot actuator in order to predict its behavior using a finite element analysis. In the first stage we are going to present a prediction of the actuator's behaviour in a specific domain using a set of boundary conditions. After that, using a generative design study we are create a stiffer structure which will improve the actuator's behaviour. Inflating behaviour mainly dependent on the material flexibility. We use the Ecoflex 30 which is a flexible material with a tensile strength of 120 psi $(0.827 \mathrm{MPa})$. The actuator diameter is $10 \mathrm{~mm}$ and is based on a rigid base. The thickness of air chamber to 0.1 $\mathrm{mm}$. The outcomes from the first FE analysis shows that the maximum displacement of the soft robot actuator is about $9 \mathrm{~mm}$ and von Mises stress is $3.83 \mathrm{MPa}$ (figure 1). The total displacement of the actuator is quite big.

In order to control the actuator behavior we are going to use a reinforcement body on the outer actuator surface, as a result of generative design. Potential solutions are outcome from the analysis. Based on one of the potential solution we design the reinforcement element (figure 2).
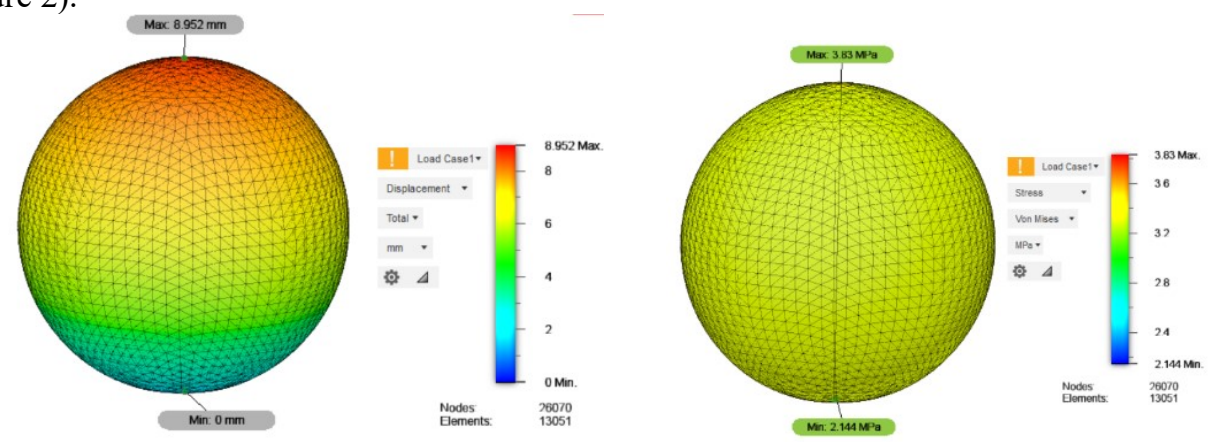

Fig. 1. Total displacement and stress results of soft robot actuator

The selected material for reinforcement element is Polylactic Acid (PLA) which is a bioplastic and common material for $3 \mathrm{~d}$ printing process. PLA can be used in soft robot actuator for surgery use. The actuator behavior changes in height during inflation $t$ at large mechanical strains. Generative design analysis starts from an initial sphere of $13 \mathrm{~mm}$ diameter and $3 \mathrm{~mm}$ thickness (figure 2). 

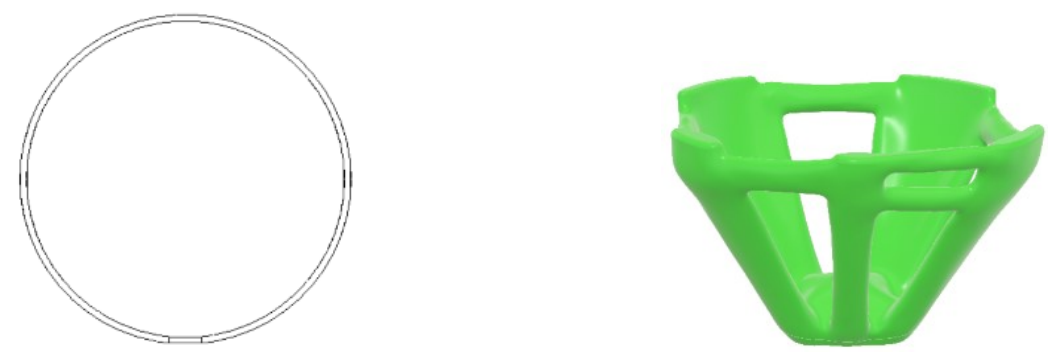

Fig. 2. In the left, the initial input geometry in GD study. In the right, the generated design reinforcement element from bioplastic material

Now the air is incoming from the base of the outer body and passes through a hole to the main actuator surface. After the generative design study is completed we verify the behavior of the reinforced structure using FEA. The tangent surfaces between the actuator and the reinforcement element are bonded. All other boundary conditions are the same as the first FE analysis. As its presented in fig. 3 the displacement of the actuator is limited, is not bigger than $2 \mathrm{~mm}$ and von Mises stress is now less than 1.733E+08 Pa.
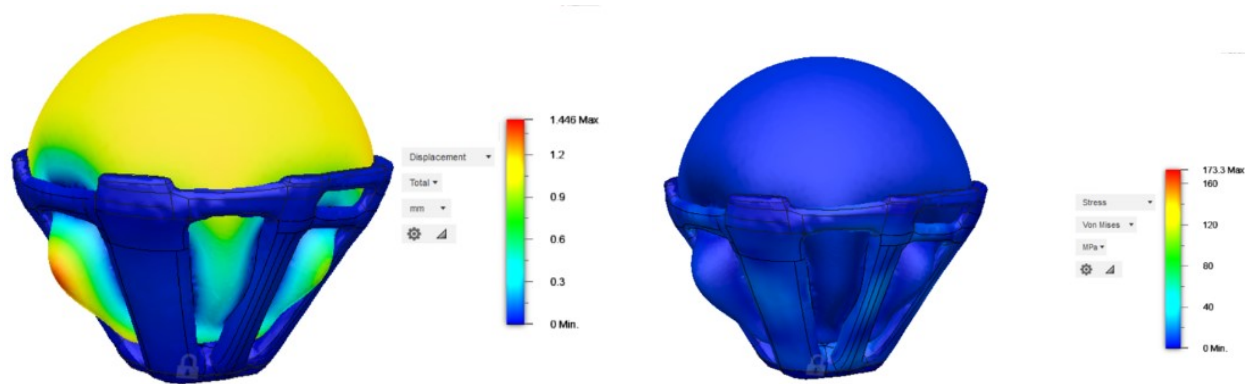

Fig. 3. Total displacement and stress results of soft robot actuator bonded with reinforcement element

\section{CONCLUSION}

Generative design is a powerful tool for designers and engineers. As methodology GD is not new, but in combination with computer raising and intelligent systems usage they become a well-developed and reliable design tool. The results of GD study are characterized by organic shapes which try to mimic the nature. Moreover, the results of a GD study can not be predicted but not exceed operational and geometric constrains. In most cases the GD 3d models are very difficult or can't be machined with traditional manufacturing methods. Additive manufacturing technology is the appropriate production method for generative design models. The generated model of soft robot actuator can be produced with a two printed head, i.e. additive manufacturing using two materials.

In the presented example, it has been demonstrated that the regenerated model limits the displacement of the soft robot actuator. The generated model is stiffer than the initial one. Further study could take into account variable (unilateral) contact effects and contact force optimization.

\section{References}

1. E. Tyflopoulos, D. Tollnes Flem, M. Steinert and A. Oslen. NordDesign, 1-24 (2018) 
2. X. Chen, Y. Tao, G. Wang, R. Kang, T. Grossman, S. Coros and S. Hudson. CHI, 1-12 (2018)

3. H. Bohnacker, B. Grob, J. Laub, and C. Lazzeroni, Generative Gestaltung (2009)

4. EOS Aerospace: https://www.eos.info/en/3d-printing-examples-applicationsinnovationstories/ruag-aerospace-3d-printed-satellite-components (2018)

5. GMmotors.https://media.gm.com/media/us/en/gm/news.detail.html/content/Pages/new s/us/en/2018/may/0503-lightweighting.html (2018)

6. Stratasys. https://3dprinting.trimech.com/case-study-fdm-bmw (2014)

7. S. Jauhar, K. Asthankar and A. Kuthe AMEA. 2181 (2012)

8. Center FM Ford. https://www.plasticstoday.com/content/ford-3d-printed-auto-partssave-millions-boost-quality/97214356120060 (2014)

9. M. Tomlin and J. Meyer 7th Altair CAE technology, 1-9 (2011)

10. C. Majidi, soft robotics 1, 5-11(2013)

11. R. Pfeifer, H. Gravato and F. Iida Twenty-Third international joint conference on Artificial Intelligence 1-9 (2013)

12. A. Calderón, J. Ugalde, Z. Joakin, C. Juan and N. Perez-Arancibia IEEE International Conference on Robotics and Biomimetics, 31-38 (2016)

13. F. Giorgio-Serchi and Weymouth G.D. Soft Robotics: Trends, Applications and Challenges 17, 37-46 (2017)

14. K. Digumarti, A. Conn and J. Rossiter IEEE Robotics and Automation Letters, 2, 2302 , (2017)

15. R. Niiyama, X. Sun, C. Sung, B. An, D. Rus and S. Kim Pouch Soft Robotics, 2, 59-70 (2015)

16. P. Polygerinos, Z. Wang. K.C. Galloway, R. J. Wood and C. J. Walsh, RAS 73, 135-143 (2015)

17. F. Ilievski, A. Mazzeo, R. Shepherd, X. Chen and G. M. Whitesides. Angewandte C., $50,(2011)$

18. I. Ntintakis, V. Iakovakis, G. Ntalos and J. Kechagias, Current Issues in Global Furniture, 14-21 (2013)

19. G. Tairidis, Phd Thesis, https://www.didaktorika.gr/eadd/handle/10442/38484 (2016)

20. G. Tairidis, A. D. Muradova and G.E. Stavroulakis, Frontiers in Built Environment J. 5, (2019)

21. I. Ntintakis, G.E. Stavroulakis and N. Plakia, SIM-AM 2019, 225-236 (2019)

22. A. W. Gebisa and H. G. Lemu, MESIC 2017, 724-731 (2017)

23. M.P. Bendsoe and O. Sigmund, Topology Optimization (2013)

24. N. Kaminakis, https://www.didaktorika.gr/eadd/handle/10442/36368 (2015)

25. N. Kaminakis, T. Drosopoulos and G.E. Stavroulakis, Arch Appl Mech 85, 1289-1306 (2015). https://doi.org/10.1007/s00419-014-0970-7 